remission at week $10 \quad(p<0.05)$. Higher proportions of patients treated with either dose of FIL than PBO achieved MCS response at week 10. At week 58, higher proportions of bio-naïve and experienced responders, and bio-experienced responders with $\geq 2$ biologic or 2 MoA failures treated with maintenance FIL $200 \mathrm{mg}$ than PBO achieved clinical remission $(p<0.05)$. Higher proportions of responders treated with maintenance FIL $200 \mathrm{mg}$ than PBO achieved MCS response at week 58 .

Conclusion FIL $200 \mathrm{mg}$ was effective in inducing and maintaining clinical remission in bio-naïve and -experienced patients. Induction results suggest FIL $200 \mathrm{mg}$ is most effective in bio-naïve patients, and those who switch after failure of 1 biologic or MoA.

\section{OMO-3 SAFETY ANALYSIS OF FILGOTINIB FOR ULCERATIVE COLITIS: PHASE 2B/3 SELECTION STUDY AND LONG- TERM EXTENSION RESULTS}

${ }^{1}$ Ben Morison*, ${ }^{2}$ Stefan Schreiber, ${ }^{3}$ Mamoru Watanabe, ${ }^{4}$ Chohee Yun, ${ }^{4}$ Yan Zhou, ${ }^{4}$ Sally Zhao, ${ }^{4}$ Jeremy Hsieh, ${ }^{5}$ Ulrik Moerch, ${ }^{6}$ Gerhard Rogler, ${ }^{7}$ Edward V Loftus. ${ }^{1}$ Norfolk and Norwich University Hospital, Norwich, UK; ${ }^{2}$ University Hospital Schleswig-Holstein, Kiel, Germany; ${ }^{3}$ Tokyo Medical and Dental University, Tokyo, Japan; ${ }^{4}$ Gilead Sciences, Inc., Foster City, USA; ${ }^{5}$ Gilead Sciences, Inc., Copenhagen, Denmark; ${ }^{6}$ University Hospital of Zurich, University of Zurich, Switzerland; ' Mayo Clinic College of Medicine, Rochester, USA

\subsection{6/gutjnl-2021-BSG.10}

Introduction Filgotinib (FIL) is an oral preferential Janus kinase (JAK) 1 inhibitor. FIL for the treatment of moderately to severely active ulcerative colitis (UC) was evaluated in the phase 2b/3, double-blind, placebo (PBO)-controlled SELECTION study (NCT02914522) and its long-term extension (LTE) study (NCT02914535). Here we report safety results.

Methods Patients received FIL $100 \mathrm{mg}$, FIL $200 \mathrm{mg}$ or PBO (2:2:1) once daily orally for up to 11 weeks for induction (cohort 1). At week 11, FIL induction responders were rerandomised 2:1 to continue FIL or receive $\mathrm{PBO}$ maintenance for 47 weeks (cohort 2). Week 10 non-responders and patients with worsening disease during the maintenance study were eligible for open-label FIL in the LTE. Patients completing the maintenance study could continue blinded dosing in the LTE. Cohort 3 comprised cohorts 1 and 2 and the LTE. Exposure-adjusted incidence rates (EAIRs) and exposureadjusted event rates (EAERs) per 100 patient-years (PYs) were calculated for treatment-emergent adverse events (AEs).

Results In cohort 1, 1069 patients received FIL and 279 patients received $\mathrm{PBO}$; baseline characteristics were generally similar across treatment groups. EAIRs for AEs of interest were similar across treatment groups in cohorts 1 and 2 . Treatment exposure for PBO, FIL $100 \mathrm{mg}$ or FIL $200 \mathrm{mg}$ in cohort 3 (i.e. cohorts $1+2+$ the LTE) was 318, 360 and 1207 PYs, and median treatment duration was 12, 11 and 67 weeks, respectively. One case of pulmonary embolism occurred with FIL $200 \mathrm{mg}$ induction and three venous thrombosis cases occurred with PBO maintenance/LTE (cohort 3). EAERs for all infections were similar across treatment groups. Opportunistic infections were rare. EAERs for serious infections were low across treatment groups (2.2 [PBO], 3.5 [FIL $100 \mathrm{mg}$ ], 2.2 [FIL $200 \mathrm{mg}$ ). EAERs for herpes zoster (HZ) were low in all treatment groups (0.3 [PBO], 0.3 [FIL $100 \mathrm{mg}$ ], 1.8 [FIL $200 \mathrm{mg}$ ]). HZ infections were cutaneous only and only one was serious. EAIRs for all infections in cohorts 1 and 2 were generally numerically higher for both $\mathrm{PBO}$ and FIL in patients over (vs under) 65 years old and in those with (vs without) biologic treatment failure.

Conclusions FIL was well tolerated in patients with UC. Aggregation of AEs typical for pan-JAK inhibition was not observed, consistent with preferential JAK-1 inhibition with FIL.

\section{OMO-4 DEVELOPING A GENOMIC BIOMARKER OF CANCER RISK IN PATIENTS WITH UC USING UNSELECTED ENDOSCOPIC BIOPSIES}

${ }^{1,2,3}$ Ibrahim Al Bakir*, ${ }^{1,4}$ Kathleen Curtius, ${ }^{1}$ Kane Smith, ${ }^{1}$ Maja Kopczynska, ${ }^{2}$ Morgan Moorghen, ${ }^{2}$ Ailsa Hart, ${ }^{1}$ Trevor Graham. ${ }^{1}$ Barts Cancer Institute, London, UK; ${ }^{2}$ St. Mark's Hospital, Harrow, UK; ${ }^{3}$ Hillingdon Hospital, Uxbridge, UK; ${ }^{4}$ University of California, San Diego, San Diego, USA

\subsection{6/gutjnl-2021-BSG.11}

Introduction Patients with ulcerative colitis (UC) are enrolled into surveillance programs for the early detection of colorectal cancer (CRC). However, most patients under surveillance are low-risk and never progress to CRC, while a significant proportion of CRCs in UC form without a preceding confirmed diagnosis of dysplasia. High resolution chromosomal copy-number alteration (CNA) analysis of unselected formalin-fixed paraffin embedded biopsies taken at surveillance colonoscopies using low pass whole genome sequencing (lpWGS) offers an appealing approach to CRC stratification.

Methods We conducted a retrospective case-control study to compare the CNA burden in four unselected non-neoplastic left-sided colorectal biopsies from patients with E2/E3 UC derived 1-5 years prior to HGD/CRC detection (cases), with that of biopsies from patients who subsequently remained HGD/CRC-free for at least 5 years (controls). The two patient groups were matched by age, gender, duration of IBD and PSC status. lpWGS was performed using a standardised pipeline for epithelial enrichment, DNA extraction, library preparation, next generation sequencing and bioinformatic analysis. Results 476 biopsies, derived from 42 cases and 77 controls, were analysed. Nearly $80 \%$ of patients had a detectable CNA in at least one of their biopsies, with the maximal CNA

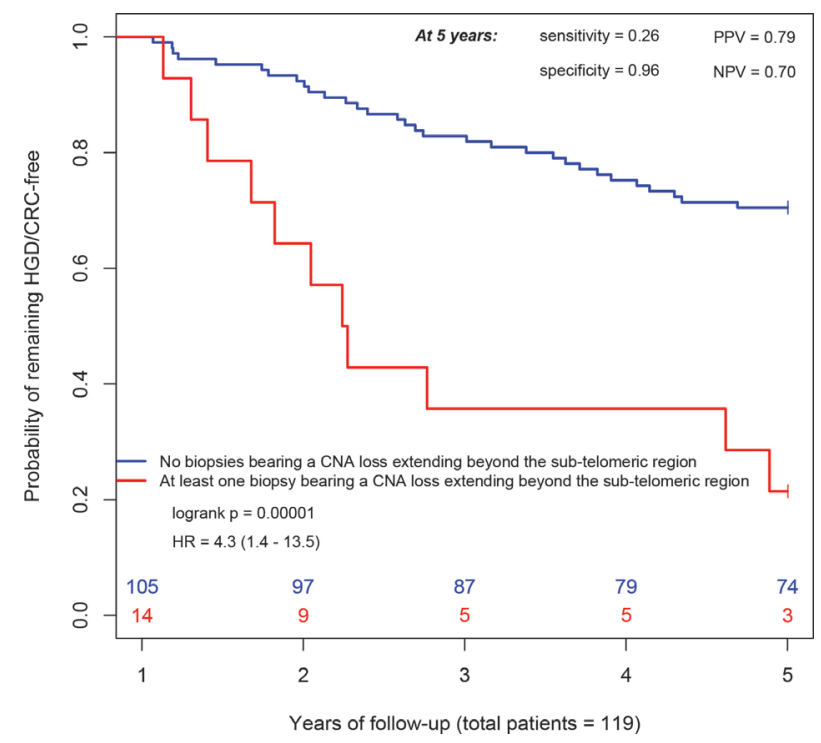

Abstract 0M0-4 Figure 1 
burden in a typical biopsy involving a median $1.1 \%$ of that biopsy's genome. The CNA burden was significantly greater in the rectum compared to the sigmoid colon and descending colon. The most common CNA events were losses of between 1-30 megabases involving the sub-telomeric regions of chromosomes 5-9 and 22, which were found in similar proportion in both case and control biopsies. However, losses extending beyond sub-telomeric regions, as well as copy number gains, were found more frequently in cases biopsies $(p<0.0001)$. The most discriminating CNA event was the presence of such a loss extending beyond subtelomeric regions in any of the patient's four biopsies (Figure 1). ROC analysis demonstrates that lpWGS output has a fair level of accuracy at predicting future $\mathrm{HGD} / \mathrm{CRC}$ risk (AUC 0.73).

Conclusions We identified multiple biopsies, predominantly in cases, with a surprisingly marked CNA burden involving over $10 \%$ of the genome, highlighting the fluid phenotype-genotype relationship. Non-dysplastic colitic epithelium can bear a significant burden of CNAs and maintain phenotypic stability for years without neoplastic transformation. Remarkably, by analysing the CNA burden of only four random biopsies, derived from less than $0.05 \%$ of the colonic surface area, we can significantly discriminate between case and control cohorts.

\section{OTH-9 EXPLORING THE IMPACT OF SHIELDING ON PATIENTS WITH INFLAMMATORY BOWEL DISEASE: A QUALITATIVE STUDY} 1,2Karen Kemp*, Pearl Avery, Lesley Dibley. 'Manchester Royal Infirmary, Manchester, UK;
${ }^{2}$ NIHR70@70, Manchester, UK

10.1136/gutjil-2021-BSG.12

Introduction In March 2020 the government introduced the concept of shielding with a new description of 'clinically extremely vulnerable' (CEV) individuals. The British Society of Gastroenterology (BSG) developed 'The Grid' stratifying patients into low, moderate and high-risk groups. We explored the experiences of patients classified as high risk who were advised to shield and patients who chose to shield.

Methods Participants were recruited from Crohn's and Colitis UK website, facebook page and newsletter, and were eligible if they shielded during any lock down period. Semi-structured interviews, held via MS Teams, Zoom or telephone between February and May 2021, were audio-recorded and transcribed professionally. Data were analysed using thematic analysis (Spencer et al.2003).

Results 27 shielding pts took part, median age 35yrs, 17 females, median disease duration $13 y r s, 16$ pts with CD, 9 pts with UC, 2 pts with IBDU. Ethnic group: White British 25; British Asian/Indian 2. BSG grid: High risk 10 pts; Moderate $16 \mathrm{pts}$, Low 1 pts. 2pts shielded due to own perception of risk to self and or family member. Four main themes emerged: Becoming CEV; IBD Preparedness; Troubled and Troublesome, and Exit strategy.

Becoming CEV patients felt 'labelled' - one day they were a normal person, the next labelled, which changed their identity. Whilst protective for some, i.e getting medication delivered, feeling 'looked after', for others this instilled fear. Conflicting information at the start of the pandemic around the need to shield caused great confusion.

IBD preparedness some patients felt that having IBD, being unwell and living through periods of isolation due to illness prepared them for shielding, having greater resilience than they thought. Many developed coping strategies, such as yoga. The positive impact of shielding was family bonding, spending unexpected time with loved ones, not needing to restrict diet on Sunday to travel to work Monday, not needing to find a toilet and overall, less stress.

Troubled and Troublesome patients reported the negative side to shielding, the loss of control, loss of identity and no separation between work and private life. Family members shielded with the patient which caused feelings of guilt and being a burden. Loss of human contact and missed life opportunities added to the anxiety. There was fear of relapse, of needing hospital appointments, and attending for infusions, and a need for more contact with their clinical team.

Exit strategy the sudden overnight end to shielding left patients feeling vulnerable, confused, and scared. Some participants have continued to shield and need further support to reconnect with normality.

Conclusion Shielding had positive and negative effects: those who had led constrained lives pre-pandemic felt prepared for shielding, others bonded with family members again, whilst some lost themselves to loneliness, overwhelming fear and anxiety. The communication around risk classification, shielding and exiting shielding could have been improved. Patients wanted more contact with healthcare professionals via a blend of remote and face to face monitoring. These lessons could inform IBD care delivery in the future.

\section{Liver}

\section{OTH-1 SARS-COV-2 INFECTION IN PATIENTS WITH AUTOIMMUNE HEPATITIS}

${ }^{1}$ Thomas Marjot*, 2,3Gustav Buescher, 2,3 Marcial Sebode, ${ }^{1}$ Eleanor Barnes, ${ }^{4}$ Alfred Barritt, ${ }^{5}$ Matthew Armstrong, ${ }^{6}$ Luke Baldelli, 'James Kennedy, ${ }^{1}$ Carolyn mercer, ${ }^{7}$ Ann-Kathrin Ozga, ${ }^{2,3}$ Christian Casar, 2,3,8 Christoph Schramm, 1,2,3 contributing Members and Collaborators of ERN RARE-LIVER/COVID-Hep/SECURE-Cirrhosis, ${ }^{4}$ Andrew Moon, ${ }^{9}$ Gwilym Webb, ${ }^{2,3}$ Ansgar Lohse. ${ }^{1}$ Oxford Liver Unit, Translational Gastroenterology Unit, Oxford Univeristy Hospitals NHS Trust, Oxford, UK; ${ }^{2}$ Department of Medicine, University Medical Centre Hamburg-Eppendorf, Germany; ${ }^{3}$ European Reference Network on Hepatological Diseases (ERN RARE-LIVER), Germany; ${ }^{4}$ Division of Gastroenterology and Hepatology, University of North Carolina, Chapel Hill, USA; ${ }^{5}$ Liver Unit, Queen Elizabeth Hospital, Birmingham, UK: ${ }^{6}$ Department of Medicine, University of North Carolina, Chapel Hill, USA; ${ }^{7}$ Institute of Medical Biometry and Epidemiology, University Medical Center Hamburg-Eppendorf, Hamburg, Germany; ${ }^{8}$ Martin Zeitz Centre for Rare Diseases, University Medical Centre Hamburg-Eppendorf, Hamburg, Germany; ${ }^{9}$ Cambridge Liver Unit, Addenbrooke's Hospital, Cambridge University Hospitals, Cambridge, UK

\subsection{6/gutjnl-2021-BSG.13}

Introduction Severe acute respiratory syndrome coronavirus 2 (SARS-CoV-2) and coronavirus disease 2019 (COVID-19) continues to have a devastating impact across the globe. However, little is known about the disease course in patients with autoimmune hepatitis (AIH).

Methods Data for patients with AIH and SARS-CoV-2 infection were combined from three international reporting registries; COVID-Hep, SECURE-cirrhosis and R-LIVER. Outcomes were compared to those with chronic liver disease of other aetiology (non-AIH CLD) and to patients without liver disease (non-CLD).

Results Between 25th March and 24th October 2020, data were collected for 932 patients with CLD and SARS-CoV-2 infection including 70 with autoimmune hepatitis (AIH). Fifty- 